\title{
The Number of Fuzzy Clopen Sets in Fuzzy Topological Spaces
}

\author{
Ali Ahmad Ali Fora* \\ Department of Mathematics, Yarmok University, Irbid, Jordan \\ *Corresponding author: afora_jo@yahoo.com
}

\begin{abstract}
We show the number of fuzzy clopen sets in an arbitrary fuzzy topological space can be any natural number greater than 1 if it is finite. We give an upper bound for this number. We shall also prove that the number of all crisp fuzzy clopen sets in an arbitrary fuzzy topological space is a power of 2 if it is finite.
\end{abstract}

Keywords: clopen, enumerating, finite set, fuzzy clopen

*This research has taken place while the author has a sabbatical leave from Yarmouk University.

Cite This Article: Ali Ahmad Ali Fora, “The Number of Fuzzy Clopen Sets in Fuzzy Topological Spaces.” Journal of Mathematical Sciences and Applications, vol. 5, no. 1 (2017): 24-26. doi: 10.12691/jmsa-5-1-4.

\section{Introduction}

After Zadeh created fuzzy sets in his classical paper [11], Chang [2] used them to introduce the concept of a fuzzy topology.

In this paper we shall follow [10] for the definitions of: fuzzy point and fuzzy topology. For instance, a fuzzy point $\mathrm{p}$ in a set $\mathrm{X}$ is a fuzzy set in $\mathrm{X}$ given by $\mathrm{p}(\mathrm{x})=\mathrm{t}$ for $\mathrm{x}=\mathrm{x}_{\mathrm{p}}(0<\mathrm{t}<1)$ and $\mathrm{p}(\mathrm{x})=0$ for $\mathrm{x} \neq \mathrm{x}_{\mathrm{p}} . \mathrm{x}_{\mathrm{p}}$ is called the support of $p$ and $p\left(x_{p}\right)=t$ the value of $p$. However, we shall agree that a fuzzy crisp point $q$ in $X$ is a fuzzy set in $X$ given by $\mathrm{q}(\mathrm{x})=1$ for $\mathrm{x}=\mathrm{x}_{\mathrm{q}}$ and $\mathrm{q}(\mathrm{x})=0$ for $\mathrm{x} \neq \mathrm{x}_{\mathrm{q}}$. It is clear that $\mathrm{p}$ is a fuzzy singleton (as introduced by Goguen [5] and used by Ghanim, Kerre and Mashhour [4]) if and only if $\mathrm{p}$ is either a fuzzy point or a fuzzy crisp point. We shall also follow [4] for the definition of $\lambda^{c}, \operatorname{Co}_{\mathrm{M}} \lambda=\lambda^{c}$ $\cap \chi_{M}$ (where $\chi_{M}$ denotes the characteristic function of M) and fuzzy subspace topology. We shall follow [8] for the definition of 'belonging to'. Namely: A fuzzy point $\mathrm{p}$ in $\mathrm{X}$ is said to belong to a fuzzy set $\lambda$ in $\mathrm{X}$ (notation: $\mathrm{p} \in \lambda)$ iff $\mathrm{p}\left(\mathrm{x}_{\mathrm{p}}\right)<\lambda\left(\mathrm{x}_{\mathrm{p}}\right)$. Finally, two fuzzy points $\mathrm{p}$ and $\mathrm{q}$ in $\mathrm{X}$ are said to be distinct iff their supports are distinct, i.e., $\mathrm{x}_{\mathrm{p}} \neq \mathrm{x}_{\mathrm{q}}$. For an arbitrary fuzzy set $\lambda$ on $\mathrm{X}$, the ordinary set $\{\mu: \mu$ is a fuzzy set on $\mathrm{X}$ and $\mu \subseteq \lambda\}$ is called the fuzzy power class of $\lambda$ and is denoted by $\mathrm{P}_{f}(\lambda)$.

Remember that a fuzzy set $\lambda$ on $\mathrm{X}$ is just a function from $X$ to the unit interval $[0,1]$. The complement of the fuzzy set $\lambda$ (denoted by $\lambda^{c}$ ) in $\mathrm{X}$ given by $\lambda^{c}(\mathrm{x})=1-\lambda$ $(x), x \in X$. If $r \in[0,1]$, then $r$ will denote the fuzzy constant set on $\mathrm{X}$ given by $\mathrm{r}(\mathrm{x})=\mathrm{r}, \mathrm{x} \in \mathrm{X}$. A fuzzy topology $\tau$ on a nonempty set $\mathrm{X}$ is a collection of fuzzy sets on $\mathrm{X}$ that 0,1 and closed under finite intersection and arbitrary union. Elements of the fuzzy topology are called fuzzy open sets and their complements are called fuzzy closed sets. A fuzzy set $\lambda$ is called fuzzy clopen set in $X$ if it is fuzzy open and fuzzy closed, simultaneously. By a fuzzy crisp set we shall mean a fuzzy set on $\mathrm{X}$ whose range is a subset of the ordinary doubleton $\{0,1\}$. (i.e., if it is a characteristic function).

Recall that a topology $\mathrm{T}$ on a nonempty set $\mathrm{X}$ is a subset of the ordinary power set of $\mathrm{X}(\mathrm{P}(X))$ that contain $\varphi$ and $X$, and is closed under arbitrary union and finite intersection. A topology on $\mathrm{X}$ is a sublattice of $(\mathrm{P}(X), \subseteq)$ with the maximum element $X$, denoted by 1 , and the minimum element $\varphi$, denoted by 0 . A set $H \subseteq \mathrm{X}$ is called an open set in the topological space $(X, T)$ provided $H \in T$. Complement of open set $\mathrm{s}$ are called closed sets. A set $\mathrm{M}$ is called a clopen set in (X,T) provided $\left\{M, M^{c}\right\} \subseteq T$ ( i.e., $\mathrm{M}$ is closed and open, simultaneously). The collection of all (fuzzy) clopen sets in (X, T) ( $\mathrm{T}$ is either a topology or a fuzzy topology on $\mathrm{X}$ ) will be denoted by $\mathrm{CO}(\mathrm{X}, \mathrm{T}$ ) (or $\mathrm{CO}(\mathrm{X})$ if no confusion will arise). The cardinality of a set $\mathrm{X}$ will be denoted by card (X) (or $|X|$ ).

$\mathrm{N}, \mathrm{Q}, \mathrm{R}$ denote the sets of natural, rational and real numbers, respectively. $T_{\text {ind }}, T_{\text {dis }}, T_{\text {sor }}$ will denote the indiscrete, the discrete and the Sorgenfrey topologies, respectively.

In a topological space $(\mathrm{X}, \mathrm{T})$, the complement of a clopen set is another clopen set (disjoint with it). Thus the number of clopen sets in any toplogical space must be even if finite. However this even number cannot be 6 for example. Fora [3] proved the following results.

1.1 THEOREM. Let $(X, T)$ be a topological space such that $\mathrm{CO}(\mathrm{X}, \mathrm{T})$ is finite. Then card $\mathrm{CO}(\mathrm{X}, \mathrm{T})=2^{\mathrm{k}}$ for some $\mathrm{k} \in \mathrm{N}$.

1.2 THEOREM. Given a nonempty set $X$ and $n \geq 1$ is any cardinal number. If $|X| \geq \mathrm{n}$, then there exists a topology $\mathrm{T}$ on $\mathrm{X}$ such that card $(\mathrm{CO}(\mathrm{X}, \mathrm{T}))=2^{\mathrm{n}}$.

One application of (fuzzy) clopen sets is that they can be used to describe (fuzzy) connectedness. In particular, a 
(fuzzy) topological space (X,T) is (fuzzy) connected if and only if card $(\mathrm{CO}(\mathrm{X}, \mathrm{T}))=2$. It is clear that in general $\mathrm{CO}(\mathrm{X}, \mathrm{T})$ need not be a topological space even if $(\mathrm{X}, \mathrm{T})$ is 0-dimensional (i.e., $\mathrm{T}$ has a base consisting of clopen sets). Indeed, $\mathrm{CO}\left(\mathrm{R}, \mathrm{T}_{\mathrm{Sorg}}\right)$ is not a topology on $\mathrm{R}$ because $(0,1)$ is not a clopen set in $\left(R, T_{\text {Sorg }}\right)$ although $(0,1)=$ $\bigcup_{n \in N}[1 / n, 1)$.

Several researchers have enumerated the topologies on a finite set $[1,6,7]$. Others have studied the number of open sets of finite topologies [9]. Fora [3] has studied the number of clopen sets of arbitrary topological space. He used an algebraic approach for his goal.

\section{Enumeration of $\mathrm{CO}(\mathrm{X}, \mathrm{T})$ for Fuzzy Topological Space $(\mathrm{X}, \mathrm{T})$}

The following result shows that the number of fuzzy clopen sets in a fuzzy topological space may be any natural number (odd as well as even) greater than 1 .

2.1 PROPOSITION. For any ordinary nonempty set $X$ and any natural number $n$, let $T=\{j / n: j=0,1,2, \ldots, n-1, n\}$. Then $(\mathrm{X}, \mathrm{T})$ is a fuzzy topological space with $\mathrm{CO}(\mathrm{X}, \mathrm{T})=\mathrm{T}$ of cardinality $n+1 \geq 2$ may be even as well as odd.

It is clear that the fuzzy constant set $\lambda=\mathrm{j} / \mathrm{n}$ is clopen in $(\mathrm{X}, \mathrm{T})$ because $\lambda^{\mathrm{c}}=(\mathrm{n}-\mathrm{j}) / \mathrm{n} \in \mathrm{T}(0 \leq j \leq n, 0 \leq n-j \leq n)$.

2.2. PROPOSITION. If $(X, T)$ is a fuzzy disconnected space, then there exist nontrivial ( i.e., $\neq 0,1$ ) fuzzy clopen sets $\lambda, \eta$ on $\mathrm{X}$ such that $\eta \leq 0.5 \leq \lambda$.

Proof: Since $(X, T)$ is a fuzzy disconnected space, there exists a nontrivial fuzzy clopen set $\mu$ in X. Now taking $\eta=\mu \cap \mu^{\mathrm{c}}$ and $\lambda=\mu \cup \mu^{\mathrm{c}}$ will complete the proof.

In the above Proposition2.2, there is no guarantee that $\lambda, \eta$ are nonconstants. Indeed, taking $\mathrm{X}=[0,1]$ and $\mathrm{T}=\left\{0,1, \mu, \mu^{\mathrm{c}}, 0.25,0.75\right\}$, where $\mu(\mathrm{x})=0.25$ for $\mathrm{x} \in \in Q \cap X$ and $\mu$ (x) $=0.75$ for $\mathrm{x} \in X-Q$. Then $\eta=\mu \cap \mu^{\mathrm{c}}=0.25$ and $\lambda=\mu \cup \mu^{\mathrm{c}}=0.75$. Notice that $(\mathrm{X}, \mathrm{T})$ has six fuzzy clopen sets, two of which are nonconstants.

The following result shows that $\mathrm{CO}(\mathrm{X}, \mathrm{T})$ can be even denumerable as well as having cardinality the continuum c. 2.3. PROPOSITION. For any ordinary nonempty set $X$, let i) $T=\{1 / n: n \in N\} \bigcup\{1-(1 / n): n \in N\}$ (ordinary union).

ii) $\tau=\{\mathrm{c}: 0 \leq c \leq 1\}$.

Then $(\mathrm{X}, \mathrm{T}),(\mathrm{X}, \tau)$ are fuzzy topological spaces satisfying the conclusion (i.e., $\mathrm{CO}(\mathrm{X}, \mathrm{T})=\mathrm{T}$ is denumerable and $\operatorname{card}(\mathrm{CO}(\mathrm{X}, \tau))=\operatorname{card}(\tau)=\mathrm{c}$.

The following result gives an upper bound for card $\mathrm{CO}(\mathrm{X}, \mathrm{T})$ for fuzzy topological space $(\mathrm{X}, \mathrm{T})$. Of course its lower bound is 2. Its value is exactly 2 for connected fuzzy topological space (X,T).

2.4. PROPOSITION. Let $X$ be an infinite set and $T$ be a fuzzy topology on $\mathrm{X}$. Then $2 \leq \operatorname{card}(\mathrm{CO}(\mathrm{X}, \mathrm{T})) \leq 2^{|X|}$.

Notice that we have used the fact that $\left|[0,1]^{X}\right|=c^{|X|}=2^{|X| \aleph_{0}}=2^{|X|}$ for $X$ an infinite set.

The following result shows that the number of crisp fuzzy clopen sets obeys the order obtained by Fora [3].
Namely, we have the following convergence result with ordinary topological spaces.

2.5. THEOREM. Let $(\mathrm{X}, \mathrm{T})$ be a fuzzy topological space such that $\mathrm{CO}(\mathrm{X}, \mathrm{T})$ is finite. Then the collection of all crisp fuzzy clopen sets in $(\mathrm{X}, \mathrm{T})$ has cardinality $2^{\mathrm{n}}$ for some natural number $\mathrm{n}$.

Proof. Denote the collection of all crisp fuzzy clopen sets in $(\mathrm{X}, \mathrm{T})$ by $\mathrm{K}(\mathrm{X}, \mathrm{T})$. Then

$$
\mathrm{K}(\mathrm{X}, \mathrm{T})=\left\{\begin{array}{l}
\mu: \mu \in T \text { and } \mu^{c} \in T \\
\text { and } \mu=\chi_{A} \text { for some } A \subseteq X
\end{array}\right\} .
$$

For $\alpha, \beta \in \mathrm{K}(\mathrm{X}, \mathrm{T})$, define the binary operation $*$ by $\alpha * \beta=\chi_{A-B} \cap \chi_{B-A}$, (where $\mathrm{A}=\alpha^{-1}(\{1\})$ and $\mathrm{B}$ $\left.=\beta^{-1}(\{1\})\right)$.

This operation makes $(\mathrm{K}(\mathrm{X}, \mathrm{T}), *)$ an abelian group with the identity element 0 . Moreover, for any $\alpha \in$ $\mathrm{K}(\mathrm{X}, \mathrm{T})$, we have $\alpha * \alpha=\chi_{A-A} \cap \chi_{A-A}=0$ (where $\mathrm{A}=$ $\left.\alpha^{-1}(\{1\})\right)$. I.e., each element has its own inverse. Thus applying the same technique as in Fora [3], we get $(\mathrm{K}(\mathrm{X}, \mathrm{T}), *) \approx$ (isomorphic to) $\mathrm{C}_{1} \mathrm{x} \mathrm{C}_{2} \mathrm{x} \ldots \mathrm{x} \mathrm{C}_{\mathrm{n}}$, where $\mathrm{C}_{\mathrm{i}} \approx \mathrm{Z}_{2}$ for $\mathrm{i}=1, \ldots, \mathrm{n}$. Hence card $\mathrm{K}(\mathrm{X}, \mathrm{T})=$ card $\left(\mathrm{C}_{1} \times \mathrm{C}_{2} \mathrm{x} \ldots \mathrm{xC_{n }}\right)=2^{\mathrm{n}}$.

Another topological PROOF. Denote the collection of all crisp fuzzy clopen sets in $(\mathrm{X}, \mathrm{T})$ by $\mathrm{K}(\mathrm{X}, \mathrm{T})$. Then

$$
\mathrm{K}(\mathrm{X}, \mathrm{T})=\left\{\begin{array}{l}
\mu: \mu \in T \text { and } \mu^{c} \in T \\
\text { and } \mu=\chi_{\mathrm{A}} \text { for some } A \subseteq X
\end{array}\right\} .
$$

Define $\tau=\left\{\mu^{-1}(\{1\}): \mu \in \mathrm{K}(\mathrm{X}, \mathrm{T})\right\}$. Then $\tau$ is indeed a topology on $\mathrm{X}$ because $\mathrm{CO}(\mathrm{X}, \mathrm{T})$ is finite. Moreover $\mathrm{CO}(\mathrm{X}, \tau)$ is finite. Hence by Theorem 1.1 (see Fora [3]). Card $(\mathrm{CO}(\mathrm{X}, \tau)))=2^{\mathrm{n}}$ for some $\mathrm{n} \in N$. Henceforth Card $\mathrm{K}(\mathrm{X}, \mathrm{T})=\operatorname{Card}(\mathrm{CO}(\mathrm{X}, \tau)))=2^{\mathrm{n}}$.

\section{Final Conclusion}

As we have discovered in [3] that in any topological space, the number of clopen sets must be a power of 2 if it is finite. Although the collection of all clopen sets in general does not form a topology. However, if the number of all clopen sets is finite then these particular sets will indeed forms a topology. The case diverse in fuzzy topological spaces. As we have pointed out, the number of fuzzy clopen sets can be any natural number greater than 1 (even or odd). It also can be denumerable or continuum. However, the collection of all crisp fuzzy clopen sets must obey the rule as in ordinary topology, i.e., in the sense that their number is a power of 2 if it is finite.

\section{Acknowledgements}

The author thanks Yarmouk University for being one of its members since 1976 and offering him a sabbatical leave this year in which this paper and other research papers were done.. I also thank Dr. Mourad Massadeh for his help in typing and rearranging this paper. I also thank my sons Dr. Ahmad Fora and Mr. Mohammad Fora for their help in the final steps of preparing and publishing the 
paper. I also thank this JOURNAL for being patient with me.

\section{References}

[1] M. Benoumhani, The number of topologies on a finite set, $J$. of Integer Sequences 9(2006) Article 06.2.6, 1-9.

[2] C.L. Chang, Fuzzy topological spaces, J. Math. Anal. Appl. 24(1968). 182-190.

[3] A. A. Fora, Algebraic Approach to the number of clopen sets in topological spaces, Bull. Cal. Math. Soc., 107, (2), (2015) 171-178.

[4] M.H. Ghanim, E.E. Kerre and A.S. Mashhour, Separation axioms, subspaces and sums in fuzzy topology, J.Math. Anal. Appl. 102 (1984) 189-202.
[5] J.A. Goguen, L-fuzzy sets, J. Math. Anal. Appl. 18 (1967) 145-174.

[6] M. Kolli, Direct and Elementary Approach to Enumerate Topologies on a Finite Set, J. of Integer Sequences 10(2007) Article 07.3.1, 1-11.

[7] H. Sharp, Jr., Cardinality of finite topologies, J. Combinatorial Theory 5(1968) 82-86.

[8] R. Srivastava, S.N.Lal and A.K. Srivastava, Fuzzy Hausdorff topological spaces, J. Math. Anal. Appl. 81(1981) 497-506.

[9] R.P. Stanley, On the number of open sets of finite topologies, $J$. Combinatorial Theory 10(1971) 74-79.

[10] C.K. Wong, Fuzzy points and local properties of fuzzy topology, J.Math. Anal. Appl. 46(1974) 316-328.

[11] L.A. Zadeh, Fuzzy sets, Inform. and Control 8(1965) 338-353. 